\title{
Finite element simulation and microstructure analysis of nickel based alloy for additive manufacturing
}

\author{
Liu Heping ${ }^{12, a}$, Sun Fenger ${ }^{1}$, Gao Yibo ${ }^{1}$, Cheng Shaolei ${ }^{1}$, and Liu Bin ${ }^{1}$ \\ ${ }^{1}$ School of Materials Science and Engineering, North University of China, Taiyuan 030051, China \\ ${ }^{2}$ School of Materials Science and Engineering, Taiyuan University of Technology, Taiyuan 030024, China
}

\begin{abstract}
In this paper, the finite element simulation of GH4169 high temperature alloy by selective laser melting was carried out, and the microstructure was analyzed by experiments. The results show that the shape of the temperature field cloud formed by the laser heat source is different from the shape of the theoretical model, but is in the shape of the ellipse. The temperature gradient at the front end of the molten pool is larger than that of the back end of the molten pool, and the isotherm of the front end of the molten pool is more intensive. The temperature of the substrate is less affected by the temperature gradient. The temperature gradient of the front end of the melting pool is larger than the back end of the molten pool, and the temperature field of selective laser melting is like a meteor with trailing tail. In the laser heat source, the temperature isotherm is the most dense and the temperature gradient is maximum. The relative effect of mechanical properties of $\delta$ phase is very complex. When the phase is precipitated by widmanstatten structure, it is easy to produce stress concentration as a source of cracks
\end{abstract}

\section{Introduction}

Ni based superalloys have high oxidation resistance, high temperature resistance, hot corrosion resistance and fatigue resistance. It has been widely used in anti-aircraft engine components, such as turbine blades and combustors, which work for a long time in high temperature environment. For the manufacture of superalloys, the methods of casting, powder metallurgy and precision machining are mainly used at present[1-5]. The cost is high and the cycle is long in the process of part design and die manufacturing. In order to further meet the requirements of material properties in aviation field, alloying elements with different contents are often added into materials. This will make the material composition more and more complex and the hot processing performance will be worse. Moreover, traditional casting methods can cause severe component segregation, which will lead to unstable microstructure and properties. With higher requirements for service performance, powder metallurgy technology can be gradually applied to aeroengine turbine blades, turbine discs and other high temperature parts. The parts formed by powder metallurgy have fine grains and uniform structure, but there are some defects such as heterogeneous inclusions, thermal induced voids and original grain boundary. Therefore, materials researchers have been studying that the only way to develop superalloys is to combine them with additive manufacturing technology[6-9].
Additive manufacturing technology combines computer-aided design, material processing and forming technology. Based on digital model files, special metal materials, non-metal materials and medical biomaterials are stacked layer by layer through software and numerical control system to manufacture physical objects. In recent years, this technology has become the mainstream direction of manufacturing technology development because of its advantages of fast research and development speed, low cost risk and high flexibility.

In this paper, GH4169 alloy powder is taken as the research object, and the effect of technological parameters on the formability of nickel-based alloy material is analyzed.

\section{Experiment and simulation method}

\subsection{Experimental method}

Nickel based alloy powder GH4169 was used as melting material with 250 mesh. Ball milling parameters are ball milling ratio $10: 1$, speed $140 \mathrm{r} / \mathrm{min}$ and ball milling $2 \mathrm{~h}$. The laser wavelength is $1.06 \mathrm{um}$, the minimum spot diameter of laser beam $0.15 \mathrm{~mm}$, and the thickness of metal powder $0.05-0.30 \mathrm{~mm}$.

\subsection{Simulation method}

In the transient temperature field, the temperature is not only related to the three-dimensional change of the space, but also a function of time. In the two-dimensional

\footnotetext{
$\overline{{ }^{a} \text { Corresponding author: peace666 } @ 126 . c o m ~}$
} 
transient temperature field, the finite element method usually divides the three-dimensional model into several finite elements to solve the instantaneous temperature distribution. This method improves the efficiency of temperature field analysis. Using the mathematical calculation model in the selected cell, the node temperature $T_{i}$ is the discrete unit[10-13].

$$
T=T=\sum_{i=1}^{n_{e}} N_{i}(x, y) T_{i}(t)
$$

where, $\mathrm{T}$ is a node temperature array and a reciprocal array for node temperature to time.

The heat conduction model of each unit is calculated as follows:

$$
K_{i j}^{e}=\int_{\Omega^{e}}\left(k_{x} \frac{\partial N_{i}}{\partial x} \frac{\partial N_{j}}{\partial x}+k_{y} \frac{\partial N_{i}}{\partial y} \frac{\partial N_{j}}{\partial y}\right) d \Omega
$$

The model calculation formula of heat transfer boundary condition of unit body can be expressed as:

$$
H_{i j}^{e}=\int_{\Theta_{3}^{e}} h N_{i} N_{j} d \Theta
$$

The specific heat capacity model of the unit is:

$$
C_{i j}^{e}=\int_{\Omega^{e}} \rho c N_{i} N_{j} d \Omega
$$

In the temperature field simulation, the temperature of the element node is the instantaneous temperature at that time, that is:

$$
T_{i}=T_{i} \quad(i=1,2,3, \ldots n)
$$

\section{Results and discussion}

\subsection{Temperature field analysis of single channel scanning process}

In the process of laser melting experiment, single-pass scanning is the basic prerequisite for optimizing process parameters. The quality of scanning line will affect the quality and performance of the whole forming workpiece. In order to reduce the number of tests and save costs, the finite element simulation method is proposed to be used to effectively select process parameters.
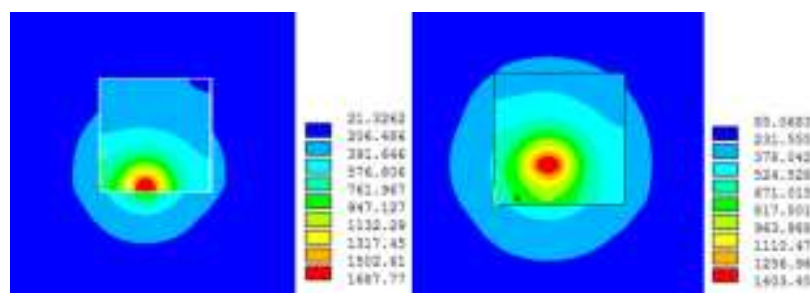

Figure 1 Temperature distribution at different time points during single channel scanning (a) $0.015 \mathrm{~s} \mathrm{(b)} 1.1 \mathrm{~s}$

Figure 1 (a) shows the first step of $0.015 \mathrm{~s}$ single-pass scanning, the temperature distribution cloud of alloy powder at the beginning of melting. As can be seen from the figure, the maximum temperature of the spot center is $1687.77{ }^{\circ} \mathrm{C}$, reaching the melting point of the alloy powder, and the powder can be melted. Figure 1 (b) is a cloud map of temperature distribution at $1.1 \mathrm{~s}$. It can be seen that the maximum temperature of the spot center dropped to $1403.45^{\circ} \mathrm{C}$ at this time. From the temperature field distribution, it can be clearly concluded that the substrate is less affected by the heat, and the basic temperature is too high to affect the powder bed. The temperature isotherm of the rear end of the molten pool is sparse, and the temperature isotherm of the front of the pool is dense. The pool temperature and the size of the molten pool gradually increase with the increase of absorbed heat.
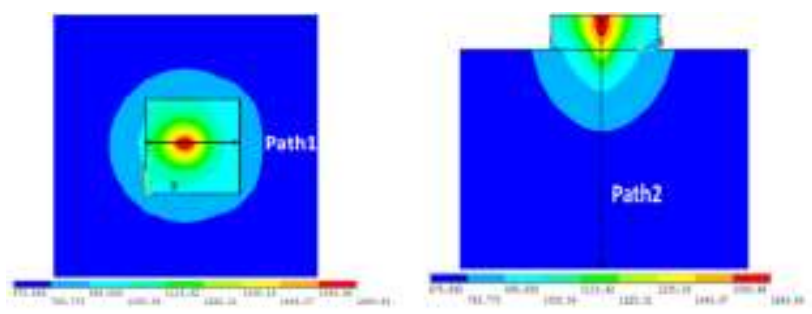

Figure 2 The temperature field distribution in single channel scanning at $0.15 \mathrm{~s}$ (a) 10 th substep (b) Cross section along the $\mathrm{X}$ axis

Figure 2(a) is a temperature field distribution cloud diagram from the single pass scanning to the 10th substep $0.15 \mathrm{~s}$. Figure 2 (b) is a temperature field distribution diagram of the molten pool along the $\mathrm{X}$-axis transverse section at that time. As can be seen from the figure, the temperature gradient at the front end of the molten pool is larger than the temperature gradient at the rear end of the molten pool. Moreover, the isotherms at the front end of the molten pool are denser. The main reason for this phenomenon is that the maximum temperature reaches $1,664.84^{\circ} \mathrm{C}$ due to the action of the heat source. The temperature exceeds the melting point of the alloy powder. The powder area is now completely melted. However, the substrate and ambient temperature are low and forced cooling occurs. Therefore, a large temperature gradient is generated As the heat is transferred, the temperature of the substrate and the surrounding environment will gradually increase. In turn, the heat affected zone is gradually enlarged.

\subsection{Temperature distribution at the end of each scan}

Figure 3 is an isotherm diagram at the end of the melting and sintering of each sintering line in a single layer scan. As can be seen from the figure, the maximum temperature at the end of the first scan line can reach 1829.26 ${ }^{\circ} \mathrm{C}$. At the end of the second scan line, the maximum temperature is $1524.73{ }^{\circ} \mathrm{C}$. The maximum temperature at the end of the third scan line is $1494.63{ }^{\circ} \mathrm{C}$. At the end of the fourth scan line, the maximum temperature is $1602.96{ }^{\circ} \mathrm{C}$. At the end of the fifth scan line, the maximum temperature is $1608.18{ }^{\circ} \mathrm{C}$. The maximum temperature at the end of the sixth scan line is $1988.45^{\circ} \mathrm{C}$. The heat generated by the laser heat source during the first pass of sintering has a greater effect on the powder. However, the influence on the substrate is small. As the laser heat source moves gradually, the scan 
line will gradually increase. The accumulation of heat causes the temperature of the powder bed to rise. Further, the temperature of the substrate is further increased. The temperature change is generally stable throughout the single layer scan. There were no high or low temperatures during the period. Because the latent heat of phase change of powder melting is considered in the simulation design. This avoids the heat accumulation effect causing the substrate temperature to be too high to affect the formation of the alloy powder. From the above analysis, it can be seen that under the action of the laser heat source. The laser heat source has a certain preheating function for the area around the powder bed. However, the thermal impact on the substrate is not very large. At the same time, the temperature isotherm at the action of the laser heat source is the most dense, that is, the temperature gradient is the largest.
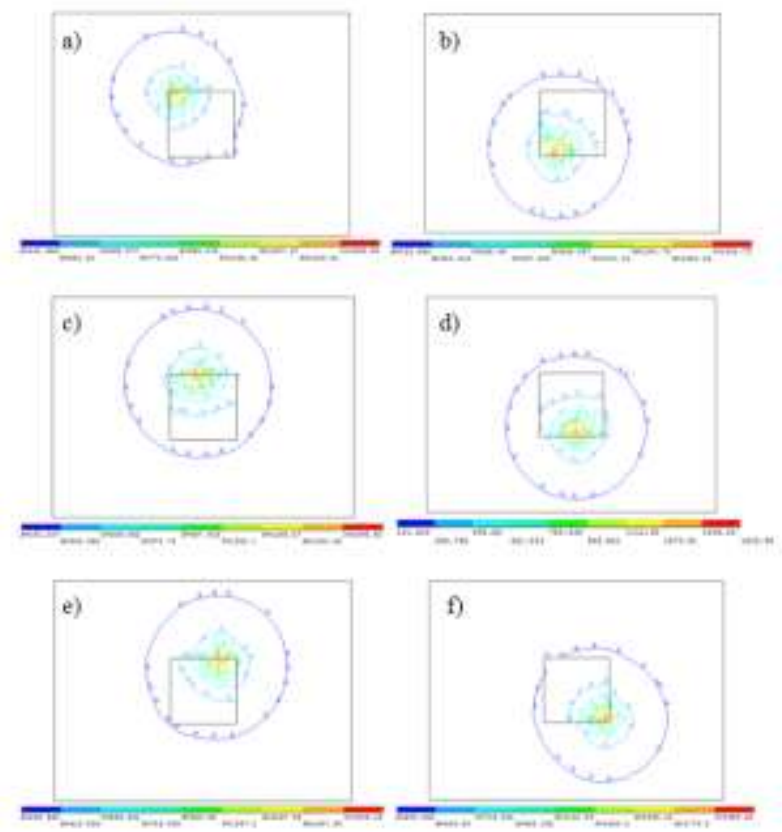

Figure 3 Isotherm diagram at the end of each scan(a)0.27s (b) $0.55 \mathrm{~s}(\mathrm{c}) 0.83 \mathrm{~s} \mathrm{(d)} 1.10 \mathrm{~s}$ (e) $1.38 \mathrm{~s}(\mathrm{f}) 1.66 \mathrm{~s}$

\subsection{Temperature changes in the whole multi- slice scanning time}

Figure 4 is a temperature change curve over time in the whole multi-layer scanning. In the experiment, the midpoint of each scan line on each layer was taken as a feature point analysis. First, the temperature distribution is divided into three stages. Each stage corresponds to the scan time period of each layer. The temperature trends of the six points in each layer are similar and cyclical. At the same time, the number of peaks per layer is the same. The characteristic point is that the maximum temperature is gradually increased. This is mainly due to the effect of heat accumulation during the forming process. However, the temperature of the entire block forming process is relatively stable. This ensures that the performance of each layer remains substantially the same during the multi-layer scanning process.
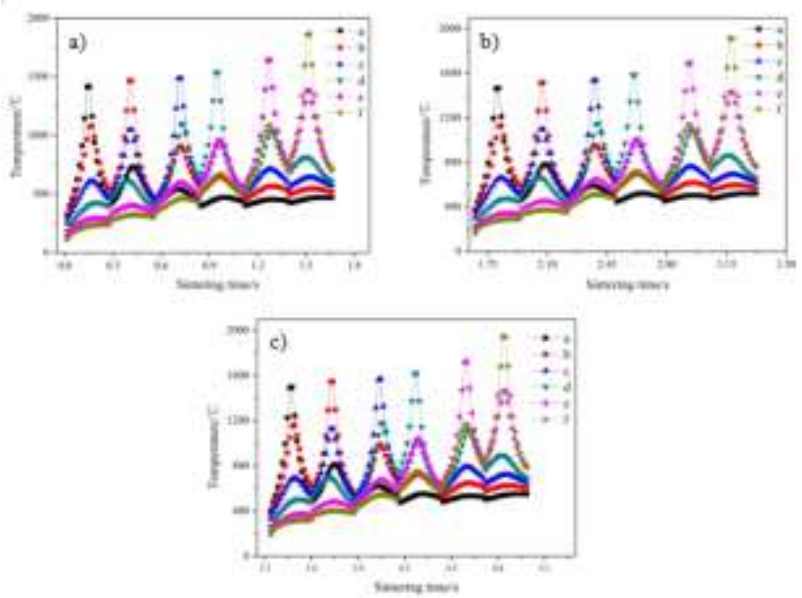

Figure 4 The temperature-time history curve in multi layer scanning $(\mathrm{V}=150 \mathrm{~mm} / \mathrm{min}, \mathrm{P}=150 \mathrm{~W}, \Psi=33 \%)$

\subsection{Microstructure analysis}
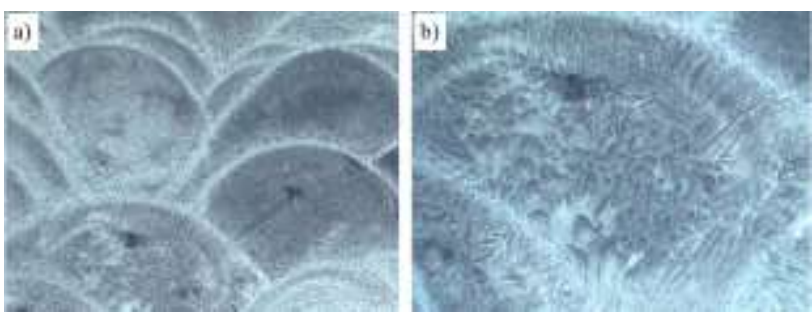

Figure 5 The morphology of nickel based alloy (a)Macroscopic morphology,(b)Local amplification

Figure 5 shows the weld pool morphology of Ni based alloy. In the experiment, the thickness of powder laying is $0.2 \mathrm{~mm}$, the width of molten pool measured 0.072-0.12 $\mathrm{mm}$, and the average thickness $0.065-0.10 \mathrm{~mm}$. The results show that the overlap degree between adjacent molten pools greatly affects the density of the formed parts. The density of formed specimens will also increase with the decrease of scanning spacing, but too small scanning spacing will cause warping and cracking of formed parts.
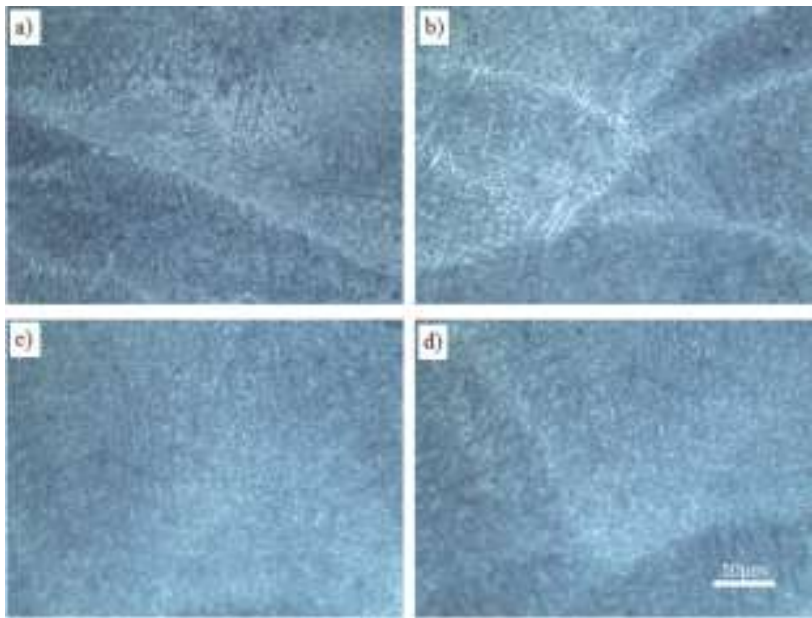

Figure 6 Microstructure of different parts of forming parts 
Figure 6 is the microstructure of different parts of the melding parts. The lighter colour is where the cladding layers overlap each other, and the area of the structure is similar to the raft structure of single crystal nickel-based alloys. The microstructure of each position is very compact. In general, GH4169 alloy precipitates $\delta$ phase, $\gamma^{\prime \prime}$ phase and $\gamma^{\prime}$ phase after solid solution and aging heat treatment $[14,15]$. Among them, the $\gamma^{\prime \prime}$ and $\gamma^{\prime}$ phases maintain a coherent relationship with the matrix, mainly through the coherent strain to strengthen the alloy. The high volume fraction of the $\gamma^{\prime \prime}$ phase is the first condition to ensure high strength of the alloy. The effect of $\delta$ phase on the relative mechanical properties is very complicated. When the $\delta$ phase is precipitated in a large amount in the Wei's structure, it is easy to cause stress concentration to become a crack source. At the same time, the content of the substrate $\mathrm{Nb}$ is reduced. The $\gamma^{\prime \prime}$ phase cannot be precipitated around it, and the strength of the alloy is lowered. However, when a proper amount of delta phase distributes along grain boundaries, the stress state at the front of the crack will be relaxed. The formation and propagation of the crack will be delayed, and the durability of the crack will be improved.

\section{Conclusion}

Laser power and scanning speed are the main parameters that affect temperature changes. The size of the bath is proportional to the instantaneous maximum temperature and laser power. But it is inversely proportional to the scanning speed. The shape of the temperature field cloud formed by the action of the laser heat source is different from the shape of the theoretical model, but is in an elliptical state. The temperature gradient at the front end of the bath is greater than the temperature gradient at the back end of the bath. Moreover, the isotherms at the front end of the molten pool are denser.

The substrate is less affected by the temperature. A large temperature gradient is formed at the laser scanning. The temperature gradient at the front of the bath is greater than the back end of the bath. The laser forming temperature field distribution is like a meteor with a tail. The temperature isotherm at the action of the laser heat source is the densest, that is, the temperature gradient is the largest. The characteristic point is that the maximum temperature is gradually increased. This is mainly due to the effect of heat accumulation during the forming process. The temperature variation over the entire multilayer scan time history is characterized by a gradual increase in the maximum temperature. This is mainly due to the effect of heat accumulation during the forming process.

The degree of overlap between adjacent molten pools greatly affects the density of the molded parts. The density of the molded part also gradually increases as the scanning pitch decreases. However, too small scanning distance will cause warping and cracking of molding parts.

\section{Acknowledgments}

This research work was financially funded by the Science and Technology Innovation Project of Shanxi Province (No.2016156), the Postdoctoral Science Foundation of China (No.2016M590214), and the Natural Science Foundation of Shanxi Province, China (No. 2015011036).

\section{References}

1. Ruiz-Sabariego J A, Pommier S. International Journal of Fatigue, 31,11 (2017)

2. Rai R K, Sahu J K. Materials Letters, 220.(2018)

3. Hosseini S A, Abbasi S M, Madar K Z, et al. Materials Chemistry \& Physics, , 211 (2018)

4. Lin Y C, Nong F Q, Chen X M, et al. Vacuum, 137(2017)

5. Shi $\mathrm{X}$, Jin $\mathrm{X}$, Lin $\mathrm{H}$, et al. Journal of Alloys \& Compounds, 693( 2017)

6. Gibson I, Rosen D W, Stucker B. Springer US, ( 2010$)$

7. Kruth J P, Leu M C, Nakagawa T. CIRP Annals Manufacturing Technology, 47,2(1998)

8. Li W, Yang K, Yin S, et al. Journal of Materials Science \& Technology, 3(2018)

9. Song J, Chew Y, Bi G, et al. Materials \& Design, 137(2018)

10. Hama $T$, Nagata $T$, Teodosiu $C$, et al. International Journal of Mechanical Sciences, 50,2 (2008)

11. Hussein A, Hao L, Yan C, et al. Materials \& Design, 52,24 (2013)

12. Gan Y, Wang W, Cui Z, et al. Optik - International Journal for Light and Electron Optics, 126,(7-8) (2015)

13. Singh S K, Mahesh K, Kumar A, et al. Materials \& Design, 31,9 (2010)

14. Li Z, Tian S, Zhao Z, et al. Journal of Aeronautical Materials, 31,5 (2011)

15. Zhang X C, Li H C, Zeng X, et al. Materials Science \& Engineering A, 682 (2017) 